\title{
The importance of research on the sense of coherence and acceptance of the disease in patients receiving palliative care due to lung cancer
}

\author{
Andrzej Nowicki* \\ Department of Oncology Nursing, Collegium Medicum in Bydgoszcz, Nicolaus Copernicus University in Toruń, Poland
}

Lung cancer is the most common malignant tumor, a huge epidemiological problem. Analyzing the mortality rate, we note that it is the most common cause of deaths in men and women. Lung cancer are diagnosed at an early stage when it can undergo surgical treatment and achieve the best results in the few cases only. In the majority of patient's lung cancer is in advanced stage at the time of diagnosis and palliative care is required in many patients. Chemotherapy should be used in conjunction with early palliative care. The main goal of care is to reduce the burden of disease, improve quality of life, relief from pain and other symptoms associated with the disease.

Holistic patient care also includes psycho-oncologic concept of treatment that has been developed and scientifically confirmed in the last 20 years. The range of activities includes a psychological counseling, supportive care and accompanying psychotherapeutic treatment of particular problems.

In the course of the disease dysfunctions of mental feeling may occur. In order, usually emotional disorders are manifested (anxiety, depression) which should be interpreted as a process of managing the disease by individual and the reactions and the limitations resulting from the health state.

Not every patient with lung cancer will require psycho-oncologic support. According to general estimate, around half of patients try to eliminate psychological burden themselves. Some patients need support only in certain periods of the disease, such as information about the diagnosis, recurrence, appearance of metastasis, or terminal phase of the disease.

The need for psycho-oncologic care is usually identified by the patient or by medical staff. However, there are often discrepancies between the need diagnosed by the expert and the need of the patient.

Advanced cancer is very aggravating mentally what consequently imposes on the medical staff need to take the efforts in form of adequate communication and care of patients with advanced lung cancer. Sense of coherence and acceptance of the disease play a significant role when experiencing stress by patient where cancer is a great source of stress, particularly in the palliative treatment period. Therefore, the psychological responses of patients to information about the disease are varied. Cancers affecting the main vital functions are cause of extremely negative reactions such is the case of lung cancer, which imparts a physiological process of breathing.
In case of lung cancer psychological stress consists of two sets of factors: clinical and social-personal. The first group includes late detection, the rapid course of the disease, poor prognosis and symptoms such as dyspnea, hemoptysis, or mental disorders, which are a strong stressor for the patient. Poor prognosis of the disease resulting in high mortality, high resistance to treatment, what in turn may lead to therapeutic nihilism in some doctors, manifested by reduced involvement in the treatment process. While the socio-personal factors include preference for male to alcohol abuse and low social status

People with lung cancer in stage IV and subjected to palliative chemotherapy are faced an extremely difficult situation in life, which undoubtedly creates problems of adaptation. Psychological adapting to the new situation is an important part of quality of life: it manifests itself in cognitive processes and behavior of the patient. The way in which the patient can handle cancer in the emotional level depends on many factors, one of which is the level of coherence and acceptance of the disease.

Study of sense of coherence and acceptance of the disease can expand the knowledge about the mental state of patients. Patients experience negative emotions before the process of acceptance and adaptation to cancer. The main strength of restoring mental balance is hope for recovery or in the case of patients receiving palliative care, hope to improve. The dynamics of the processes occurring in the mental sphere and the diversity of attitudes of patients in disease poses to oncologists the need to familiarize with the basic issues of psycho-oncology. In this way, the holistic care of patients suffering from lung cancer can be properly fulfilled. Understanding these issues can be translated into the daily work of the therapeutic team. The study of these variables should facilitate understanding of the needs and behaviors of patients, primarily to develop appropriate behavior and communication with patients undergoing palliative treatment and their families.

The results of studies assessing the sense of coherence and the level of acceptance of the disease may contribute to the development of models of care, including the sphere of mental health in particular.

Correspondence to: Andrzej Nowicki, Department of Oncology Nursing Collegium Medicum in Bydgoszcz, Nicolaus Copernicus University in Toruń, Poland, E-mail: anow1_xl@wp.pl

Received: January 05, 2017; Accepted: January 18, 2017; Published: January 21 2017 
In this way, we can improve the quality of medical care, supporting the development of the area of nursing psychological care of patients with lung cancer. This is a very important aspect of holistic care of patient subjected of palliative chemotherapy, which focuses on terminally ill patient and is only intended to alleviate the symptoms and, rarely, life extension. This does not, however, leads to cure cancer, what should be kept in mind the daily nursing practice.

Copyright: (2017 Nowicki A. This is an open-access article distributed under the terms of the Creative Commons Attribution License, which permits unrestricted use, distribution, and reproduction in any medium, provided the original author and source are credited. 